Supplement of Geosci. Model Dev., 11, 2739-2762, 2018

https://doi.org/10.5194/gmd-11-2739-2018-supplement

(C) Author(s) 2018. This work is distributed under

the Creative Commons Attribution 4.0 License.

(c) (1)

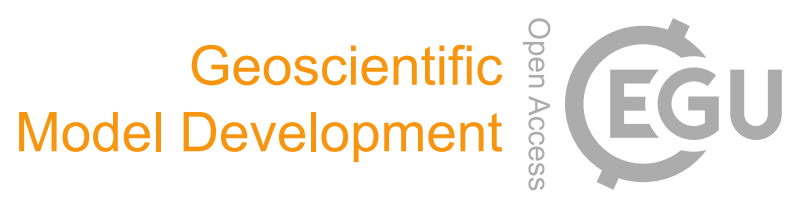

Supplement of

\title{
MOPSMAP v1.0: a versatile tool for the modeling of aerosol optical properties
}

\author{
Josef Gasteiger and Matthias Wiegner \\ Correspondence to: Josef Gasteiger (josef.gasteiger@univie.ac.at)
}

The copyright of individual parts of the supplement might differ from the CC BY 4.0 License. 


\section{Contents}

S1 Orientation averaging of irregularly-shaped particles and accuracy assessment

S1.1 Orientation averaging scheme . . . . . . . . . . . . . 2

S1.2 Accuracy assessment using dense Euler angle grid . . . . . . . . . . . . . . 3

S1.3 Accuracy assessment using spheroids . . . . . . . . . . . . . . 6

S2 Maximum TMM size parameters 7

S3 Transition from TMM to IGOM $\quad 8$

S4 Example for decomposition of a mode into contributions from data set grid points 


\section{S1 Orientation averaging of irregularly-shaped particles and accuracy assessment}

\section{S1.1 Orientation averaging scheme}

Table S1: $\beta_{e}$ and $\gamma_{e}$ grid points for irregularly-shaped particles in the MOPSMAP data set.

\begin{tabular}{|l|l|}
\hline$\beta_{e}$ & $\gamma_{e}$ \\
\hline $0^{\circ}, 180^{\circ}$ & $0^{\circ}$ \\
\hline $15^{\circ}, 165^{\circ}$ & $0^{\circ}, 60^{\circ}, 120^{\circ}, \ldots, 300^{\circ}$ \\
\hline $30^{\circ}, 150^{\circ}$ & $0^{\circ}, 30^{\circ}, 60^{\circ}, \ldots, 330^{\circ}$ \\
\hline $45^{\circ}, 60^{\circ}, 75^{\circ}, 90^{\circ}, 105^{\circ}, 120^{\circ}, 135^{\circ}$ & $0^{\circ}, 15^{\circ}, 30^{\circ}, \ldots, 345^{\circ}$ \\
\hline
\end{tabular}

The particle orientation is specified by three Euler angles $\left(\alpha_{e}, \beta_{e}, \gamma_{e}\right)$ as described by Yurkin and Hoekstra (2011). Averaging over $\beta_{e}$ is done with a step width of $15^{\circ}$, and for each $\beta_{e}$ up to $24 \gamma_{e}$ are used for averaging (see details in Table S1). The averaging over $\alpha_{e}$ is done within a single ADDA computation because rotation over $\alpha_{e}$ is equivalent to the rotation of the scattering plane and is computationally cheap. The optical properties are averaged over $32 \alpha_{e}$. In total, for a single particle 206 individual ADDA runs are performed and, if the averaging over $\alpha_{e}$ is considered, 6592 orientations are evaluated. For the numerical calculation of orientation averages of extensive optical properties $\zeta \in\left\{C_{\text {ext }}, C_{\text {sca }}, C_{\text {sca }} \cdot \mathbf{F}\right\}$, the following steps were applied.

Averaging over $\alpha_{e}$ is done by ADDA for each $\beta_{e}-\gamma_{e}$-pair using the option '-phi_integr 1 '. The $\alpha_{e}$-averaged quantity is denoted as $\zeta_{\alpha}\left(\beta_{e}, \gamma_{e}\right)$.

As the next step, averaging over $\gamma_{e}$ is done (outside ADDA) for each $\beta_{e}$ using

$$
\zeta_{\alpha, \gamma}\left(\beta_{e}\right)=\frac{1}{N_{\gamma}} \sum_{i_{\gamma}=1}^{N_{\gamma}} \zeta_{\alpha}\left(\beta_{e}, \gamma_{e}\left[i_{\gamma}\right]\right) .
$$

where $N_{\gamma}$ is the number of equidistant $\gamma_{e}$ grid points for the given $\beta_{e}$ (Table S1).

Finally, the orientation-averaged $\zeta$ is obtained by averaging over $\beta_{e}$ using

$$
\zeta=\frac{1}{2} \int_{0^{\circ}}^{180^{\circ}} \widetilde{\zeta}_{\alpha, \gamma}\left(\beta_{e}\right) \cdot \sin \beta_{e} \cdot d \beta_{e}
$$

where $\widetilde{\zeta}_{\alpha, \gamma}\left(\beta_{e}\right)$ is linearly interpolated between the available $\zeta_{\alpha, \gamma}\left(\beta_{e}\right)$ grid points (Eq. S1, Table S1). Numerical integration of Eq. S2 is performed using a step width of $\Delta \beta_{e}=$ $0.15^{\circ}$. 


\section{S1.2 Accuracy assessment using dense Euler angle grid}

Here we present a comparison of single particle properties calculated either with the orientation averaging scheme used in the MOPSMAP data set (Sect. S1.1) or using a much denser grid of orientation angles ( $5^{\circ}$ step size for $\beta_{e}$ and $\left.\gamma_{e}\right)$. This comparison provides an estimation of the accuracy of the former using the latter as reference.

Irregular shapes $\mathrm{B}, \mathrm{C}, \mathrm{D}$, and $\mathrm{F}$ with $m=1.52+0.0043 i$ at six size parameters $x_{v}$ from 10.0 to 20.8 are considered, i.e., 24 different single particles. In the following, tables are shown for the extinction efficiency $q_{\text {ext }}$ (Table S2), scattering efficiency $q_{\text {sca }}$ (Table S3), forward scattering phase function $a_{1}\left(0^{\circ}\right)$ (Table S4), backscattering phase function $a_{1}\left(180^{\circ}\right)$ (Table S5), and the normalized 2,2-element of the scattering matrix at backward direction $a_{2}\left(180^{\circ}\right) / a_{1}\left(180^{\circ}\right)$ (Table S6), where each line corresponds to a size parameter and the four values separated by slashes correspond to the different irregular shapes $\mathrm{B} / \mathrm{C} / \mathrm{D} / \mathrm{F}$.

In case of $q_{\text {ext }}$ and $q_{\text {sca }}$, i.e. properties integrated over all scattering angles, the deviation between MOPSMAP and the reference is virtually zero (Tables S2, S3). The same is true for forward scattering (Table S4). Larger deviations typically in the order of a few percent (max. 14\%) occur in case of backscattering. It is well known that scattering under $180^{\circ}$ is very sensitive to various parameters of a scattering problem, here the particle orientation. The effect on atmospheric aerosols is however reduced as under realistic conditions over- and underestimates partly compensate according to the ensemble of different particles.

Table S2: Extinction efficiency $q_{\text {ext }}$ from the MOPSMAP data set compared to results obtained using a dense grid of step size $5^{\circ}$ for $\beta_{e}$ and $\gamma_{e}$. The four values separated by slashes correspond to shapes B, C, D, and F. The relative deviation of $q_{\text {ext }}$ of MOPSMAP from the reference is rounded to full percent values.

\begin{tabular}{|l|l|l|l|}
\hline size & MOPSMAP data set & dense $\beta_{e}$ and $\gamma_{e}$ grid & rel. dev. in $\%$ \\
\hline$x_{v}=10.0$ & $2.269 / 2.064 / 2.268 / 2.069$ & $2.267 / 2.053 / 2.273 / 2.076$ & $0 /+1 / 0 / 0$ \\
\hline$x_{v}=12.0$ & $2.521 / 2.442 / 2.421 / 2.279$ & $2.530 / 2.430 / 2.424 / 2.075$ & $0 /+1 / 0 / 0$ \\
\hline$x_{v}=14.4$ & $2.180 / 2.367 / 2.294 / 2.272$ & $2.184 / 2.371 / 2.294 / 2.280$ & $0 / 0 / 0 / 0$ \\
\hline$x_{v}=17.3$ & $2.203 / 2.097 / 2.214 / 2.090$ & $2.205 / 2.100 / 2.218 / 2.101$ & $0 / 0 / 0 /-1$ \\
\hline$x_{v}=19.0$ & $2.303 / 2.200 / 2.243 / 2.106$ & $2.306 / 2.200 / 2.245 / 2.114$ & $0 / 0 / 0 / 0$ \\
\hline$x_{v}=20.8$ & $2.229 / 2.289 / 2.227 / 2.195$ & $2.232 / 2.297 / 2.230 / 2.181$ & $0 / 0 / 0 /+1$ \\
\hline
\end{tabular}


Table S3: Scattering efficiency $q_{\text {sca }}$ from the MOPSMAP data set compared to results obtained using a dense grid of step size $5^{\circ}$ for $\beta_{e}$ and $\gamma_{e}$. The four values separated by slashes correspond to shapes $\mathrm{B}, \mathrm{C}, \mathrm{D}$, and $\mathrm{F}$. The relative deviation of $q_{s c a}$ of MOPSMAP from the reference is rounded to full percent values.

\begin{tabular}{|l|l|l|l|}
\hline size & MOPSMAP data set & dense $\beta_{e}$ and $\gamma_{e}$ grid & rel. dev. in $\%$ \\
\hline$x_{v}=10.0$ & $2.076 / 1.881 / 2.101 / 1.904$ & $2.074 / 1.870 / 2.106 / 1.910$ & $0 /+1 / 0 / 0$ \\
\hline$x_{v}=12.0$ & $2.295 / 2.226 / 2.223 / 2.082$ & $2.303 / 2.214 / 2.226 / 2.075$ & $0 /+1 / 0 / 0$ \\
\hline$x_{v}=14.4$ & $1.916 / 2.114 / 2.059 / 2.042$ & $1.920 / 2.118 / 2.060 / 2.049$ & $0 / 0 / 0 / 0$ \\
\hline$x_{v}=17.3$ & $1.898 / 1.803 / 1.941 / 1.822$ & $1.899 / 1.807 / 1.944 / 1.832$ & $0 / 0 / 0 /-1$ \\
\hline$x_{v}=19.0$ & $1.975 / 1.885 / 1.948 / 1.816$ & $1.977 / 1.886 / 1.949 / 1.824$ & $0 / 0 / 0 / 0$ \\
\hline$x_{v}=20.8$ & $1.879 / 1.954 / 1.910 / 1.883$ & $1.881 / 1.961 / 1.913 / 1.869$ & $0 / 0 / 0 /+1$ \\
\hline
\end{tabular}

Table S4: Forward scattering phase function $a_{1}\left(0^{\circ}\right)$ from the MOPSMAP data set compared to results obtained using a dense grid of step size $5^{\circ}$ for $\beta_{e}$ and $\gamma_{e}$. The four values separated by slashes correspond to shapes B, C, D, and F. The relative deviation of $a_{1}\left(0^{\circ}\right)$ of MOPSMAP from the reference is rounded to full percent values.

\begin{tabular}{|l|l|l|l|}
\hline size & MOPSMAP data set & dense $\beta_{e}$ and $\gamma_{e}$ grid & rel. dev. in $\%$ \\
\hline$x_{v}=10.0$ & $74.00 / 70.20 / 82.59 / 78.66$ & $73.94 / 69.62 / 82.73 / 78.62$ & $0 /+1 / 0 / 0$ \\
\hline$x_{v}=12.0$ & $119.8 / 121.9 / 128.3 / 126.7$ & $120.3 / 121.6 / 128.4 / 126.8$ & $0 / 0 / 0 / 0$ \\
\hline$x_{v}=14.4$ & $154.7 / 176.6 / 180.0 / 188.7$ & $154.8 / 176.8 / 180.1 / 188.8$ & $0 / 0 / 0 / 0$ \\
\hline$x_{v}=17.3$ & $224.8 / 225.4 / 253.7 / 253.3$ & $224.8 / 225.5 / 253.8 / 253.2$ & $0 / 0 / 0 / 0$ \\
\hline$x_{v}=19.0$ & $288.1 / 288.4 / 312.8 / 309.4$ & $288.0 / 288.2 / 313.1 / 309.6$ & $0 / 0 / 0 / 0$ \\
\hline$x_{v}=20.8$ & $340.4 / 366.8 / 378.2 / 391.0$ & $340.7 / 367.2 / 378.5 / 389.5$ & $0 / 0 / 0 / 0$ \\
\hline
\end{tabular}

Table S5: Backward scattering phase function $a_{1}\left(180^{\circ}\right)$ from the MOPSMAP data set compared to results obtained using a dense grid of step size $5^{\circ}$ for $\beta_{e}$ and $\gamma_{e}$. The four values separated by slashes correspond to shapes B, C, D, and F. The relative deviation of $a_{1}\left(180^{\circ}\right)$ of MOPSMAP from the reference is rounded to full percent values.

\begin{tabular}{|l|l|l|l|}
\hline size & MOPSMAP data set & dense $\beta_{e}$ and $\gamma_{e}$ grid & rel. dev. in $\%$ \\
\hline$x_{v}=10.0$ & $0.3855 / 0.3277 / 0.5068 / 0.3184$ & $0.3758 / 0.3262 / 0.4960 / 0.3084$ & $+3 / 0 /+2 /+3$ \\
\hline$x_{v}=12.0$ & $0.3445 / 0.2643 / 0.4352 / 0.3270$ & $0.3238 / 0.2557 / 0.4553 / 0.3254$ & $+6 /+3 /-4 /+1$ \\
\hline$x_{v}=14.4$ & $0.3488 / 0.2409 / 0.4596 / 0.4018$ & $0.3423 / 0.2492 / 0.4648 / 0.3844$ & $+2 /-3 /-1 /+5$ \\
\hline$x_{v}=17.3$ & $0.3244 / 0.2423 / 0.4581 / 0.4634$ & $0.3091 / 0.2581 / 0.4448 / 0.4306$ & $+5 /-6 /+3 /+8$ \\
\hline$x_{v}=19.0$ & $0.2866 / 0.2200 / 0.4537 / 0.4625$ & $0.2702 / 0.2174 / 0.4367 / 0.4115$ & $+6 /+1 /+4 /+12$ \\
\hline$x_{v}=20.8$ & $0.2882 / 0.2185 / 0.4403 / 0.4352$ & $0.2800 / 0.2170 / 0.4285 / 0.3822$ & $+3 /+1 /+3 /+14$ \\
\hline
\end{tabular}


Table S6: Normalized (2,2)-element of the scattering matrix at backward direction $a_{2}\left(180^{\circ}\right) / a_{1}\left(180^{\circ}\right)$ from the MOPSMAP data set compared to results obtained using a dense grid of step size $5^{\circ}$ for $\beta_{e}$ and $\gamma_{e}$. The four values separated by slashes correspond to shapes $\mathrm{B}, \mathrm{C}, \mathrm{D}$, and $\mathrm{F}$. The relative deviation of $a_{2}\left(180^{\circ}\right) / a_{1}\left(180^{\circ}\right)$ of MOPSMAP from the reference is rounded to full percent values.

\begin{tabular}{|l|l|l|l|}
\hline size & MOPSMAP data set & dense $\beta_{e}$ and $\gamma_{e}$ grid & rel. dev. in $\%$ \\
\hline$x_{v}=10.0$ & $0.4894 / 0.5856 / 0.3478 / 0.3342$ & $0.5081 / 0.5166 / 0.3385 / 0.3347$ & $-4 /+13 /+3 / 0$ \\
\hline$x_{v}=12.0$ & $0.5398 / 0.5713 / 0.3671 / 0.3195$ & $0.5135 / 0.5407 / 0.3497 / 0.3417$ & $+5 /+6 /+5 /-6$ \\
\hline$x_{v}=14.4$ & $0.5356 / 0.5489 / 0.3493 / 0.3229$ & $0.5200 / 0.5385 / 0.3415 / 0.3396$ & $+3 /+2 /+2 /-5$ \\
\hline$x_{v}=17.3$ & $0.4884 / 0.5684 / 0.4016 / 0.3274$ & $0.4890 / 0.5454 / 0.3580 / 0.3574$ & $0 /+4 /+12 /-8$ \\
\hline$x_{v}=19.0$ & $0.4799 / 0.5362 / 0.3404 / 0.3343$ & $0.4800 / 0.5159 / 0.3630 / 0.3606$ & $0 /+4 /-6 /-7$ \\
\hline$x_{v}=20.8$ & $0.5188 / 0.5382 / 0.3838 / 0.3294$ & $0.4973 / 0.5387 / 0.3760 / 0.3590$ & $+4 / 0 /+2 /-8$ \\
\hline
\end{tabular}




\section{S1.3 Accuracy assessment using spheroids}

Here ADDA together with the orientation averaging scheme used for the irregular shapes in the MOPSMAP data set (as described in Sect. S1.1) is applied to prolate spheroids with $m=1.52+0.0043 i$ and $\epsilon^{\prime}=2.0$ (ADDA option '-shape ellipsoid 1.02 .0 '). Volumeequivalent size parameters $x_{v}=2,4$, and 10 are considered. As reference the optical properties of the same randomly-oriented particles are calculated with the TMM code of Mishchenko and Travis (1998).

The comparison again shows that the integrated parameters and the forward scattering almost perfectly agree whereas for backscattering few cases with larger deviations up to $17 \%$ are obtained. In general, the relative deviations are of similar magnitude as those found in our tests of Sect. S1.2 though the number of independent ADDA calculations is lower for spheroids than for irregular particles because of the rotation symmetry of spheroids.

Table S7: Properties of prolate spheroids with $\epsilon^{\prime}=2.0$ and $m=1.52+0.0043 i$. The three values separated by slashes correspond to size parameters $x_{v}=2,4$, and 10 . The relative deviation of ADDA from TMM is rounded to full percent values.

\begin{tabular}{|l|l|l|l|}
\hline optical parameter & ADDA + orient. avg. & TMM & rel. deviation in \% \\
\hline$q_{\text {ext }}$ & $1.656 / 3.861 / 2.274$ & $1.650 / 3.860 / 2.256$ & $0 / 0 /+1$ \\
\hline$q_{\text {sca }}$ & $1.621 / 3.780 / 2.068$ & $1.615 / 3.778 / 2.048$ & $0 / 0 /+1$ \\
\hline$a_{1}\left(0^{\circ}\right)$ & $5.741 / 18.86 / 69.30$ & $5.748 / 18.84 / 69.20$ & $0 / 0 / 0$ \\
\hline$a_{1}\left(180^{\circ}\right)$ & $0.1069 / 0.1700 / 0.3597$ & $0.0948 / 0.1686 / 0.3568$ & $+13 /+1 /+1$ \\
\hline$a_{2}\left(180^{\circ}\right) / a_{1}\left(180^{\circ}\right)$ & $0.9350 / 0.5175 / 0.4721$ & $0.9230 / 0.4927 / 0.5678$ & $+1 /+5 /-17$ \\
\hline
\end{tabular}




\section{S2 Maximum TMM size parameters}

A detailed list of maximum TMM size parameters for all 22680 combinations of refractive index and shape from the MOPSMAP spheroid data set is provided for download at https://doi.org/10.5281/zenodo.1284217 (file spheroids_tmm_max-sizes.txt). A summary is given in Table S8 for different aspect ratios $\epsilon^{\prime}$ and refractive index ranges. The TMM calculations for a given refractive index and shape (always iterating from small to large $x_{c}$ with steps of $5 \%$ ) are terminated either when a size parameter equal or larger than the minimum upper size parameter given in Table S8 is successfully modeled or if TMM does not converge. Furthermore, the results were checked for plausibility as discussed in our paper. As a consequence, the maximum TMM size parameter in the MOPSMAP data set is often 0\% - 5\% larger than given in Table S8 but can be smaller in case of numerical problems for specific shape and refractive index combinations.

Table S8: Minimum upper size parameters $x_{c}$ for the TMM calculations of oblate and prolate spheroids as function of aspect ratio $\epsilon^{\prime}$ for different refractive index ranges. For details see text.

\begin{tabular}{|l|l|l|l|l|}
\hline & $\begin{array}{l}1.28 \leq m_{r} \leq 1.6 \\
\text { and } m_{i} \leq 0.1376\end{array}$ & $\begin{array}{l}1.64 \leq m_{r} \leq 2 \\
\text { and } m_{i} \leq 0.1376\end{array}$ & $\begin{array}{l}\text { all other } m \\
\text { with } m_{r} \geq 1.0\end{array}$ & $m_{r}<1$ \\
\hline$\epsilon^{\prime}=1.2$ & 120 & 118 & 60 & 25 \\
$\epsilon^{\prime}=1.4$ & 120 & 84 & 60 & 25 \\
$\epsilon^{\prime}=1.6$ & 110 & 76.2 & 55 & 25 \\
$\epsilon^{\prime}=1.8$ & 88 & 65.8 & 44 & 25 \\
$\epsilon^{\prime}=2.0$ & 63 & 56.8 & 31.5 & 25 \\
\hline$\epsilon^{\prime}=2.2$ & 51 & 44.5 & 25.5 & 10 \\
$\epsilon^{\prime}=2.4$ & 40 & 31.6 & 20.0 & 10 \\
$\epsilon^{\prime}=2.6$ & 33 & 24.8 & 16.5 & 10 \\
$\epsilon^{\prime}=2.8$ & 29 & 21.4 & 14.5 & 10 \\
$\epsilon^{\prime}=3.0$ & 25 & 20.4 & 12.5 & 10 \\
\hline$\epsilon^{\prime}=3.4$ & 19.4 & 16.7 & 9.7 & 5 \\
$\epsilon^{\prime}=3.8$ & 16.7 & 14.5 & 8.4 & 5 \\
$\epsilon^{\prime}=4.2$ & 14.5 & 13.1 & 7.3 & 5 \\
$\epsilon^{\prime}=4.6$ & 12.5 & 11.9 & 5.3 & 5 \\
$\epsilon^{\prime}=5.0$ & 11.3 & 10.0 & & 5 \\
\hline
\end{tabular}




\section{S3 Transition from TMM to IGOM}

Figs. S1 to S8 illustrate the angle-specific scattering as function of size parameter calculated by the TMM (solid lines) and the IGOM approximation (dashed lines). The transitions between TMM and IGOM are shown as dotted lines. The upper plots are similar to those presented in Fig. 2a of Dubovik et al. (2006) (shifted by some factor) but also include results for backscattering $\left(180^{\circ}\right)$. The lower plots show the ratio between the scattering matrix elements $a_{2}$ and $a_{1}$. All plots are for $m=1.52+0.0030406 \mathrm{i}$, close to the value used by Dubovik et al. (2006), and the different plots show different particle shapes.

The figures show a very smooth transition between TMM and IGOM for the scattering intensity in the forward direction. For the backward direction some cases with jumps at the transition size parameters are found, indicating uncertainties of the IGOM approximation for the corresponding scattering angle. It is well known that backscattering is particularly difficult to model. Note that the contribution of IGOM results to the optical properties of typical dust ensembles at visible wavelengths is low so that uncertainties of ensemble backscattering properties arising from IGOM uncertainties are normally limited. 

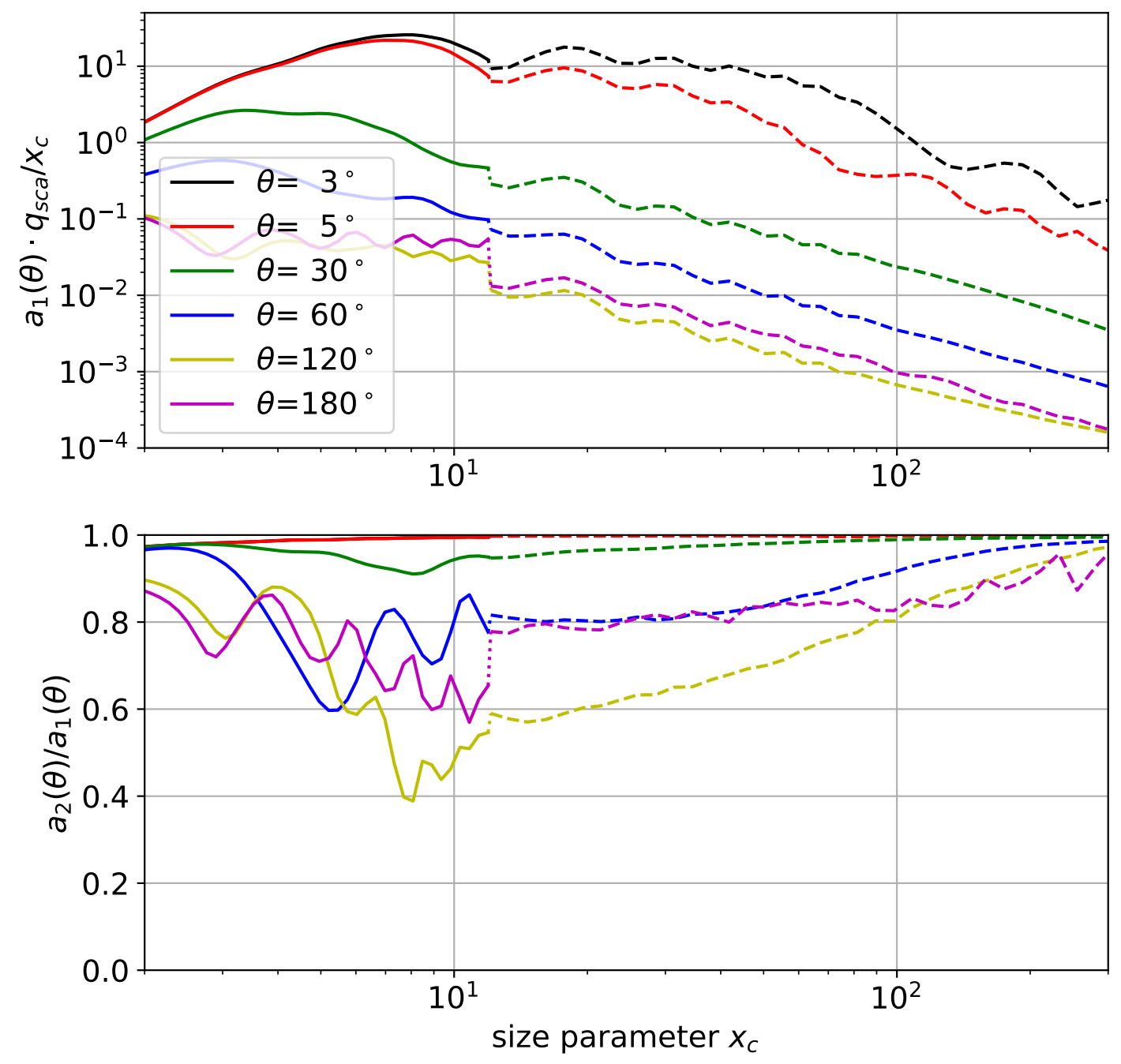

Figure S1: Comparison of IGOM (dotted lines) with TMM (solid lines) for prolate spheroids with $\epsilon^{\prime}=\mathbf{5 . 0}$ and $m=1.52+0.0030406 \mathrm{i}$ at different scattering angles $\theta$ (indicated by color). 

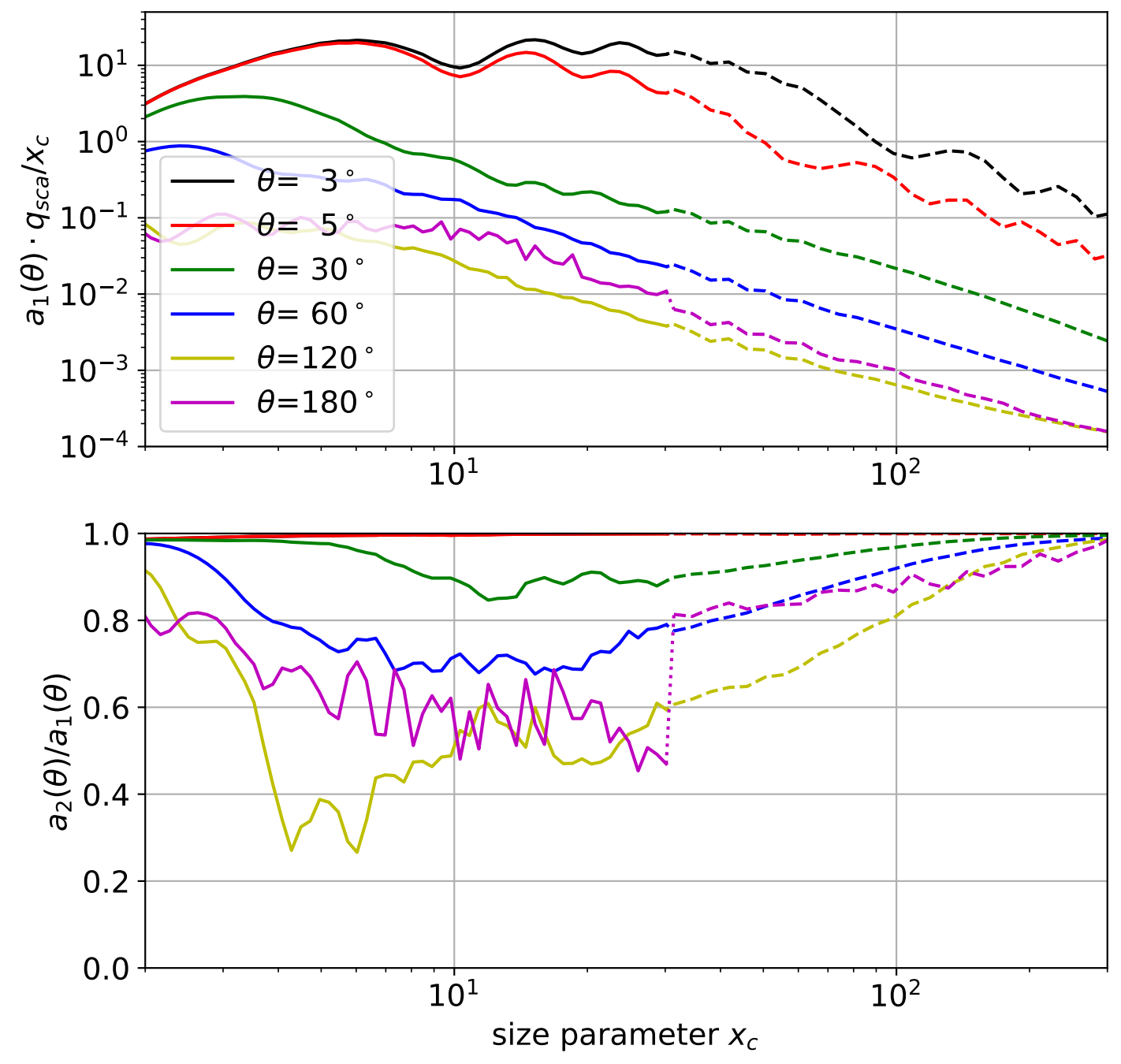

Figure S2: Comparison of IGOM (dotted lines) with TMM (solid lines) for prolate spheroids with $\epsilon^{\prime}=\mathbf{2 . 8}$ and $m=1.52+0.0030406 \mathrm{i}$ at different scattering angles $\theta$ (indicated by color). 

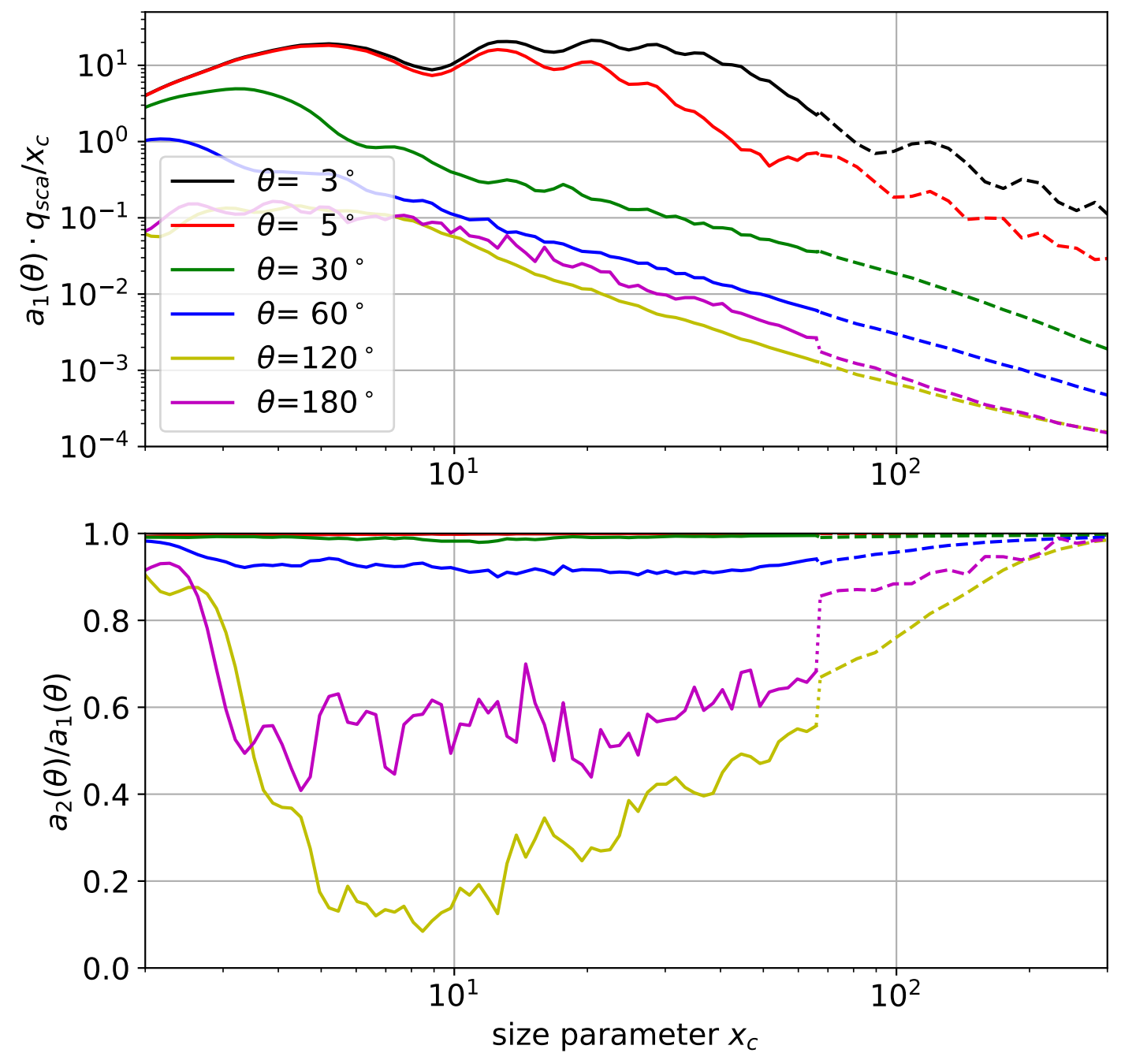

Figure S3: Comparison of IGOM (dotted lines) with TMM (solid lines) for prolate spheroids with $\epsilon^{\prime}=\mathbf{2 . 0}$ and $m=1.52+0.0030406 \mathrm{i}$ at different scattering angles $\theta$ (indicated by color). 

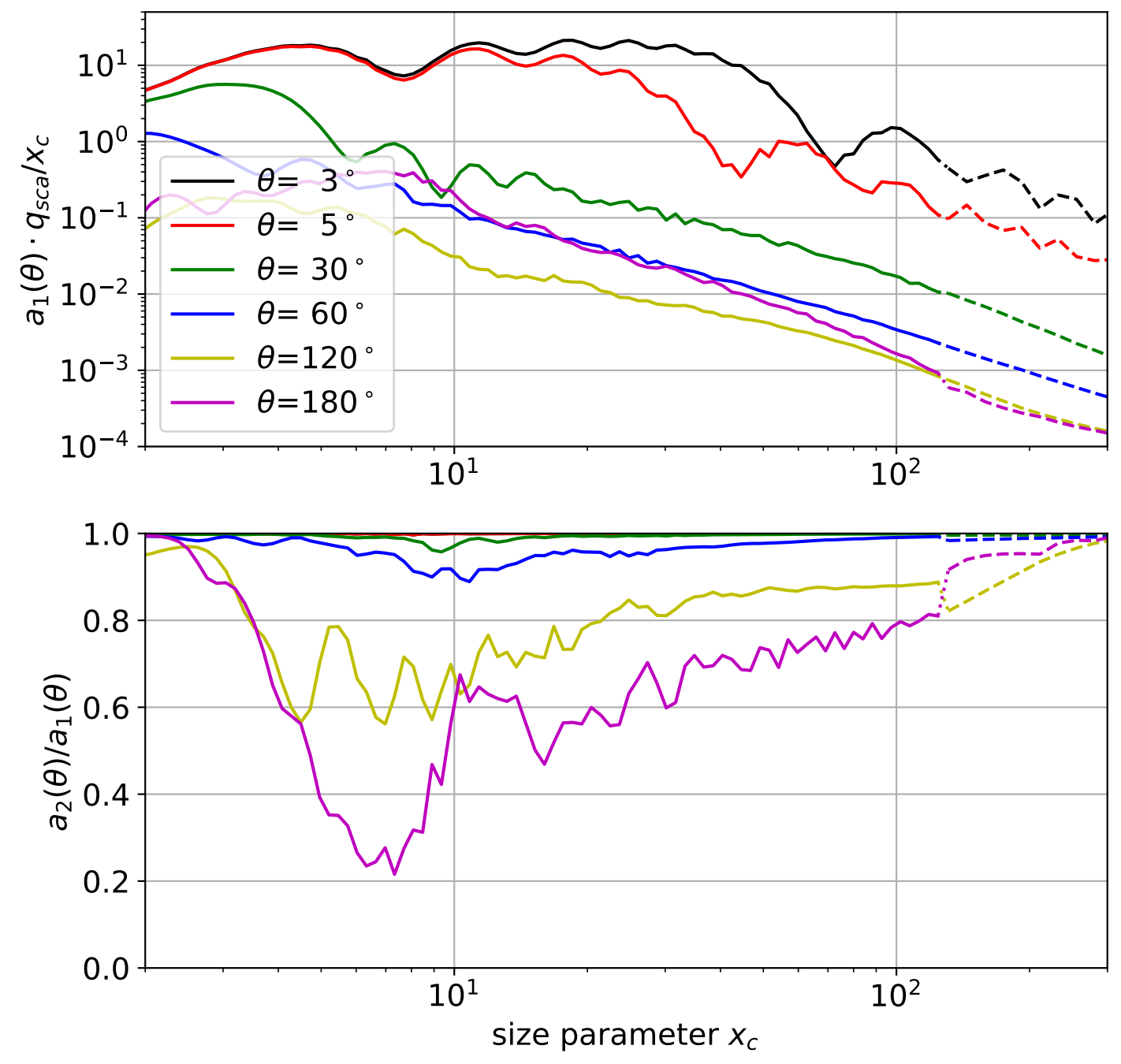

Figure S4: Comparison of IGOM (dotted lines) with TMM (solid lines) for prolate spheroids with $\epsilon^{\prime}=\mathbf{1 . 4}$ and $m=1.52+0.0030406 \mathrm{i}$ at different scattering angles $\theta$ (indicated by color). 

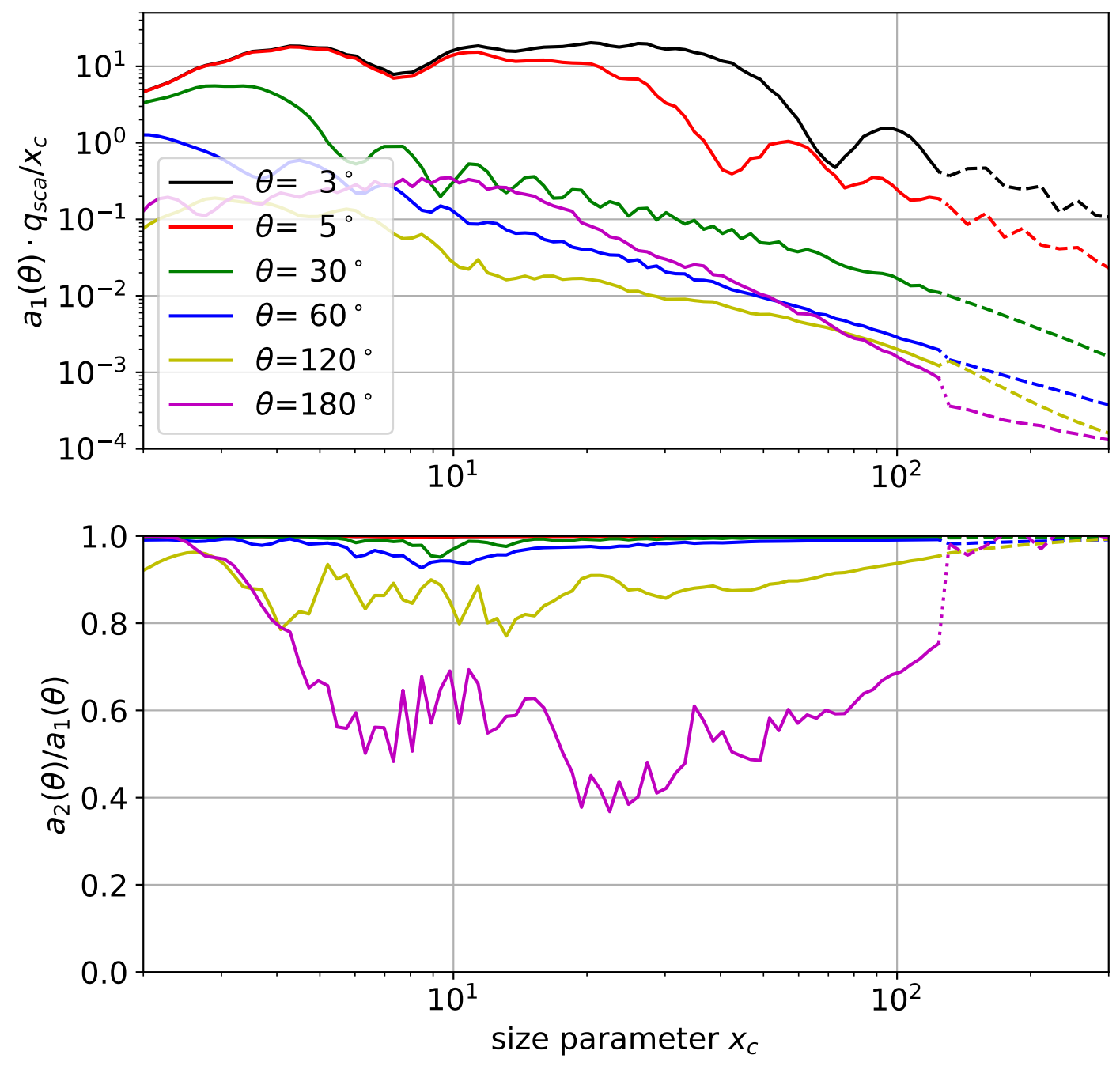

Figure S5: Comparison of IGOM (dotted lines) with TMM (solid lines) for oblate spheroids with $\epsilon^{\prime}=\mathbf{1 . 4}$ and $m=1.52+0.0030406 \mathrm{i}$ at different scattering angles $\theta$ (indicated by color). 

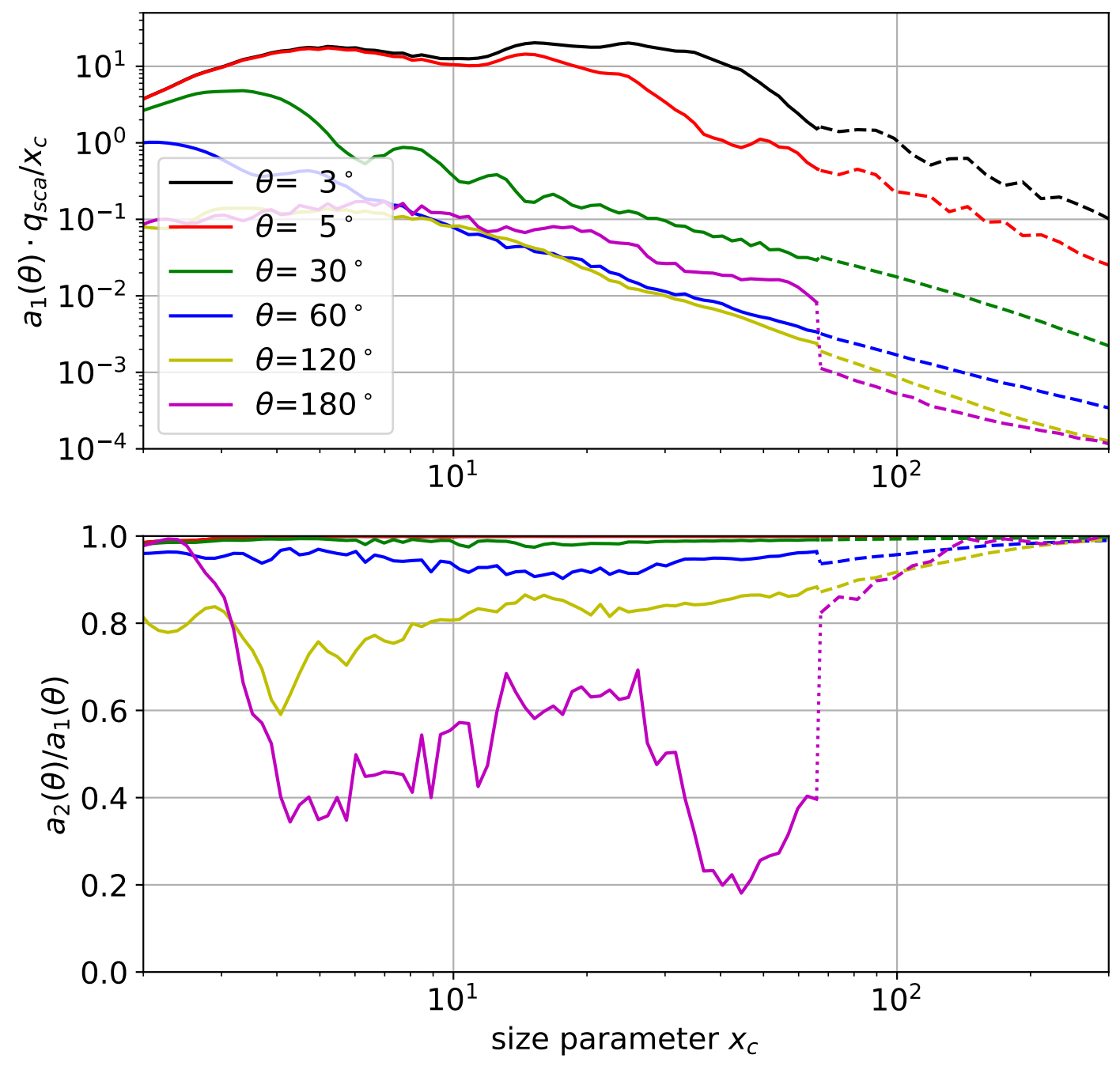

Figure S6: Comparison of IGOM (dotted lines) with TMM (solid lines) for oblate spheroids with $\epsilon^{\prime}=\mathbf{2 . 0}$ and $m=1.52+0.0030406 \mathrm{i}$ at different scattering angles $\theta$ (indicated by color). 

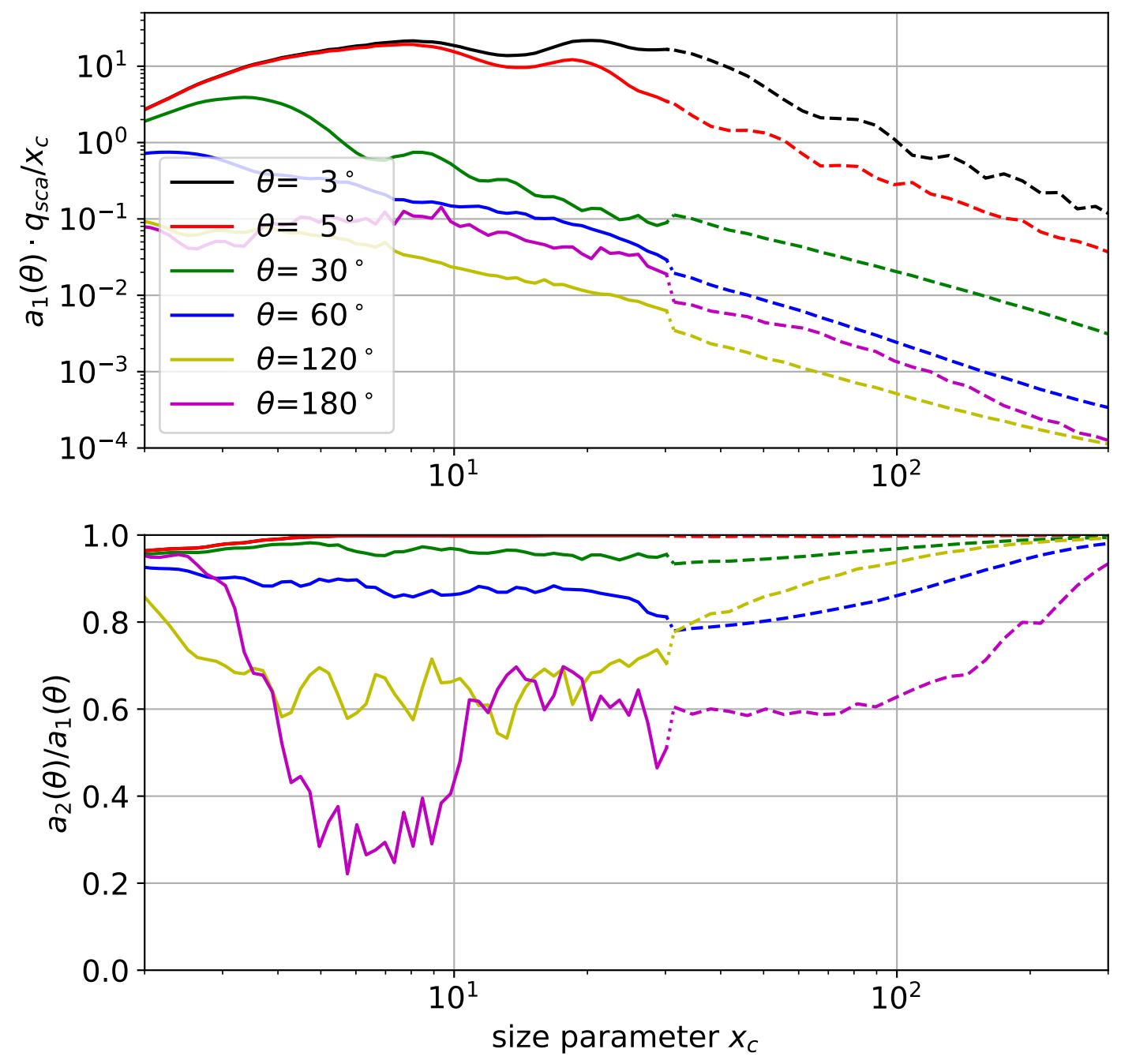

Figure S7: Comparison of IGOM (dotted lines) with TMM (solid lines) for oblate spheroids with $\epsilon^{\prime}=\mathbf{2 . 8}$ and $m=1.52+0.0030406 \mathrm{i}$ at different scattering angles $\theta$ (indicated by color). 

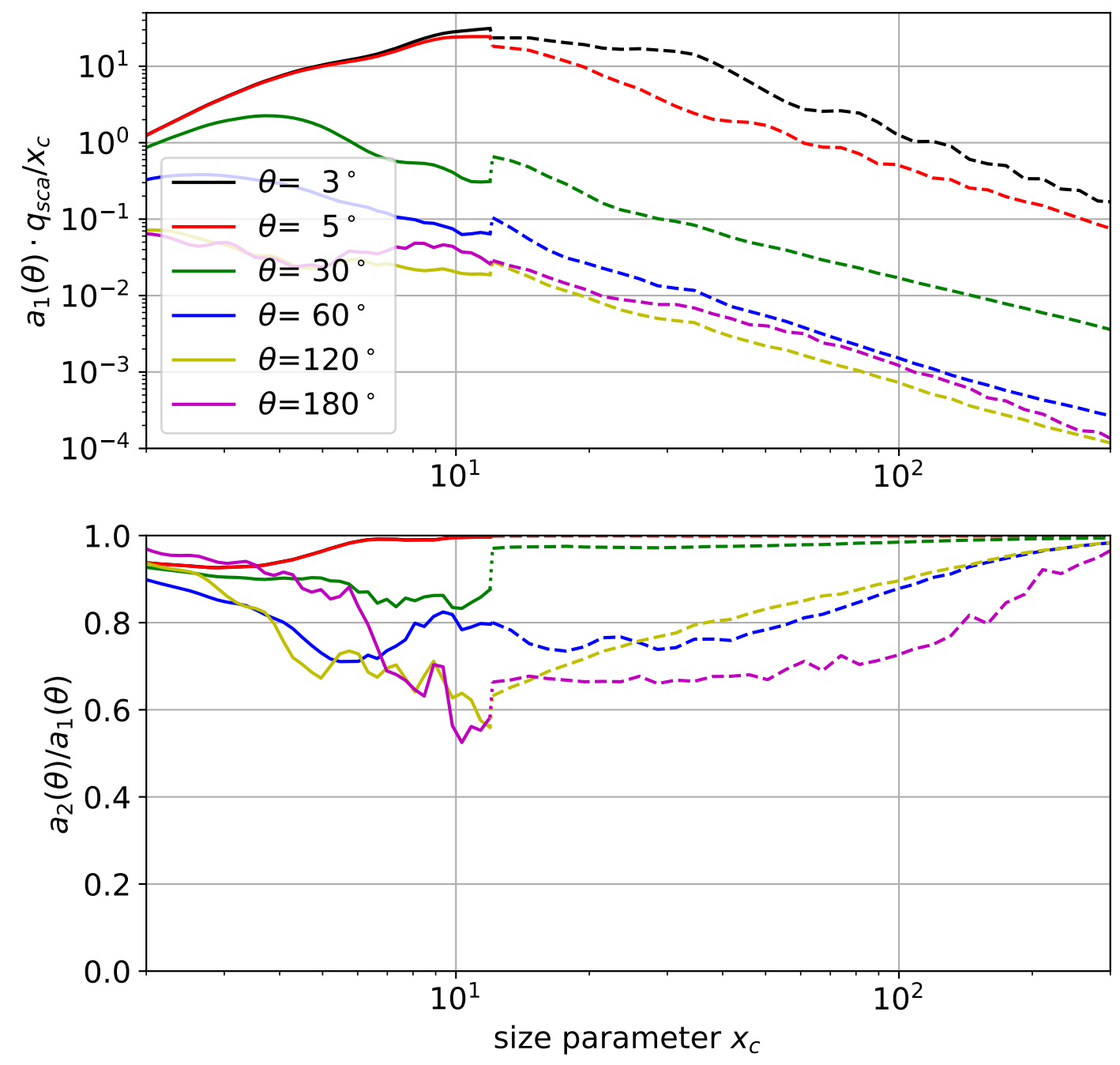

Figure S8: Comparison of IGOM (dotted lines) with TMM (solid lines) for oblate spheroids with $\epsilon^{\prime}=\mathbf{5 . 0}$ and $m=1.52+0.0030406 \mathrm{i}$ at different scattering angles $\theta$ (indicated by color). 


\section{S4 Example for decomposition of a mode into contributions from data set grid points}

Suppose, a mode has spheroids with $m_{r}=1.53, m_{i}=0$, and $\epsilon_{m}=1.92$. Then the MOPSMAP grid points of each dimension and their weights are

- $m_{r, i}=1.52$ with $w_{m_{r}, i}=0.75$

- $m_{r, i+1}=1.56$ with $w_{m_{r}, i+1}=0.25$

- $m_{i, j}=0$ with $w_{m_{i}, j}=1$

- $m_{i, j+1}=0.0005375$ with $w_{m_{i}, j+1}=0$

- $\epsilon_{m, k}=1.8$ with $w_{\epsilon_{m}, k}=0.4$

- $\epsilon_{m, k+1}=2.0$ with $w_{\epsilon_{m}, k+1}=0.6$

The contributions from the different $\left(m_{r}, m_{i}, \epsilon_{m}\right)$-grid points (i.e., netcdf files) included in the data set are therefore

1. $m_{r, i}=1.52, m_{i, j}=0, \epsilon_{m, k}=1.8$ with $w=0.75 \cdot 1 \cdot 0.4=0.30$

2. $m_{r, i}=1.52, m_{i, j}=0, \epsilon_{m, k+1}=2.0$ with $w=0.75 \cdot 1 \cdot 0.6=0.45$

3. $m_{r, i}=1.52, m_{i, j+1}=0.0005375, \epsilon_{m, k}=1.8$ with $w=0.75 \cdot 0 \cdot 0.4=0.00$

4. $m_{r, i}=1.52, m_{i, j+1}=0.0005375, \epsilon_{m, k+1}=2.0$ with $w=0.75 \cdot 0 \cdot 0.6=0.00$

5. $m_{r, i+1}=1.56, m_{i, j}=0, \epsilon_{m, k}=1.8$ with $w=0.25 \cdot 1 \cdot 0.4=0.10$

6. $m_{r, i+1}=1.56, m_{i, j}=0, \epsilon_{m, k+1}=2.0$ with $w=0.25 \cdot 1 \cdot 0.6=0.15$

7. $m_{r, i+1}=1.56, m_{i, j+1}=0.0005375, \epsilon_{m, k}=1.8$ with $w=0.25 \cdot 0 \cdot 0.4=0.00$

8. $m_{r, i+1}=1.56, m_{i, j+1}=0.0005375, \epsilon_{m, k+1}=2.0$ with $w=0.25 \cdot 0 \cdot 0.6=0.00$

The weighted sum of these 8 contributions (with 4 of them being zero) gives the optical properties of the mode. 